\title{
The distortion of a cylinder with non-uniform axial heat conduction
}

\author{
J.R. BARBER \\ Department of Mechanical Engineering and Applied Mechanics, The University of Michigan, Ann Arbor, \\ MI 48109, USA
}

(Received January 28, 1982; in revised form March 18, 1982)

\begin{abstract}
Closed form expressions are developed for the thermoelastic curvature of the initially plane end faces of a traction free cylinder subjected to arbitrary axisymmetric heat flux, the curved surfaces being assumed insulated. The solution is developed from a potential function representation of displacement and temperature for an elastic layer. The reciprocal theorem is invoked to show that the tractions at the curved surface of the cylinder vary linearly along the axis and they are removed by superposition of biaxial bending. It is found that the curvature of the plane ends depends on the local heat flux and the mean heat flux, whilst the cylindrical face distorts into a cone.
\end{abstract}

\section{Introduction}

Considerable experimental research has been directed to the resistance to heat flow across an interface between two solids, generally known as "thermal contact resistance". The most commonly used experimental system consists of two circular cylinders whose plane or slightly crowned ends are pressed together $[1,2,3]$. The opposite extremities of the cylinders are maintained at different temperatures, and care is taken to minimise heat losses from the exposed cylindrical surfaces.

It was first recognised by Clausing [4] that the thermal distortion of the cylinders has a significant influence on the contact conditions. In particular, it causes the thermal contact resistance to depend on the direction of heat flow when the materials are dissimilar. This phenomenon is known as "thermal rectification" [5,6].

In subsequent contributions to this subject, attempts have been made to assess the magnitude of the effect, using approximations to the thermoelastic distortion of the cylinders based on results for the semi-infinite solid or for uniform heat flux $[7,8]$. In this paper, we shall show that the thermal distortion of the surfaces of the cylinder can be described in closed form in terms of the heat fluxes across the ends, provided that the cylindrical faces are insulated.

\section{Statement of the problem}

We consider the cylinder defined by $0<z<\ell, r<a$ in cylindrical co-ordinates $r, \theta, z$ as shown in Fig. 1. The surface $r=a$ is assumed to be insulated, whilst on the end faces 
there is an arbitrary axisymmetric heat flux

$$
\begin{aligned}
q_{z}=-k \frac{\partial T}{\partial z} & =q_{0}(r), & & z=0 \\
& =q_{\ell}(r), & z & =\ell
\end{aligned}
$$

where $T$ is temperature and $k$ the conductivity of the material. We assume that the temperature has reached a steady state so that the total heat flux

$$
Q=2 \pi \int_{0}^{a} r q_{z}(r) \mathrm{d} r
$$

is constant for all values of $z$. Note that this imposes a constraint on the permissible boundary conditions $(1,2)$ above. The axisymmetric boundary conditions guarantee that the temperature field is axisymmetric throughout the cylinder.

All the surfaces of the cylinder are assumed to be free of tractions and the problem is to find the distorted shape of the cylinder.

\section{Solution}

We develop the solution to the problem by superposition of a particular thermoelastic solution and a corrective isothermal solution chosen to satisfy the traction free condition for the cylinder. For the particular solution we take

$$
\begin{aligned}
& u=2 k \frac{\partial \phi}{\partial z}-\nabla \phi, \\
& \alpha(1+\nu) T=\frac{\partial^{2} \phi}{\partial z^{2}}, \\
& \nabla^{2} \phi=0,
\end{aligned}
$$

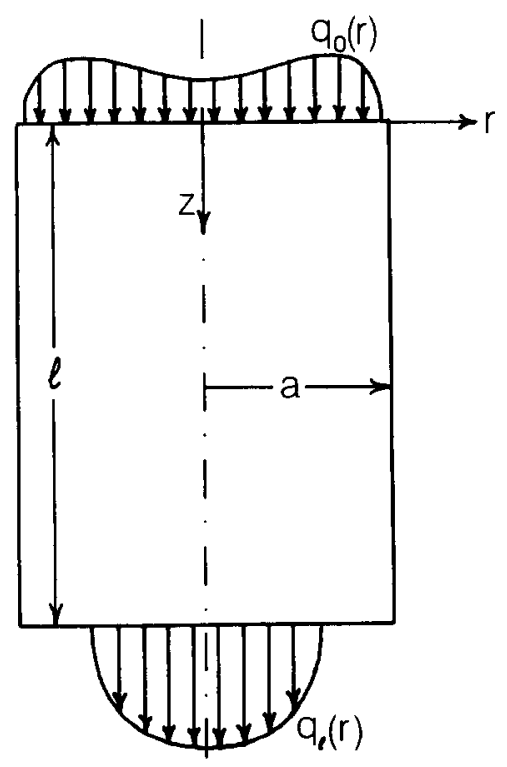

Figure 1. Geometry and boundary conditions of the problem. 
see [9], for which the stress components are

$$
\begin{aligned}
& \sigma_{r r}=\frac{2 \mu}{r} \frac{\partial \phi}{\partial r}, \quad \sigma_{\theta \theta}=2 \mu \frac{\partial^{2} \phi}{\partial r^{2}}, \\
& \sigma_{z z}=\sigma_{r \theta}=\sigma_{\theta z}=\sigma_{z r}=0,
\end{aligned}
$$

if $T$ and hence $\phi$ are independent of $\theta$.

The constants $\alpha, \mu, \nu$ are respectively the coefficient of thermal expansion, modulus of rigidity and Poisson's ratio for the material.

For this particular solution, the sum of the principal curvatures of any $z$ plane is proportional to the local heat flux $[9,10]-$-i.e.

$$
\frac{\partial^{2} u_{z}}{\partial r^{2}}+\frac{1}{r} \frac{\partial u_{z}}{\partial r}=\frac{\alpha(1+\nu) q_{z}}{k},
$$

as can be verified by substitution from Eqns. $(4,5)$ above.

If we apply this solution to the cylinder of Fig. 1, it is clear that the traction free condition will be met except for the occurrence of non-zero normal tractions $\sigma_{r r}$ on the surface $r=a$. These tractions can be related to the temperature, since from Eqns. $(5,6)$,

$$
\alpha(1+\nu) T=\frac{\partial^{2} \phi}{\partial z^{2}}=-\frac{1}{r} \frac{\partial}{\partial r} r \frac{\partial \phi}{\partial r} .
$$

Integrating Eqn. (9), we find

$$
r \frac{\partial \phi}{\partial r}=-\alpha(1+\nu) \int_{0}^{r} T(r, z) \mathrm{d} r,
$$

and hence

$$
\sigma_{r r}=-\frac{2 \alpha \mu(1+\nu)}{r^{2}} \int_{0}^{r} T(r, z) \mathrm{d} r
$$

from Eqn. (7).

\section{Use of the reciprocal theorem}

To develop an appropriate corrective solution, we first evaluate the integral in Eqn. (11) for $r=a$ by applying the reciprocal theorem to the temperature field in the cylinder. Consider the two identical cylinders shown in Figs. 2(a, b). Both cylinders are insulated on the surface $r=a$. Cylinder (a) conducts a uniform unit flux

$$
q_{z}=1,
$$

corresponding to the temperature field

$$
T=-\frac{z}{k} ;
$$

whilst cylinder (b) has an arbitrary axisymmetric temperature field with

$$
\begin{array}{llll}
q_{z}=q_{0}(r), & T=T_{0}(r) \quad \text { at } & z=0, \\
q_{z}=q_{1}(r), & T=T_{1}(r) & \text { at } & z=z_{1} .
\end{array}
$$




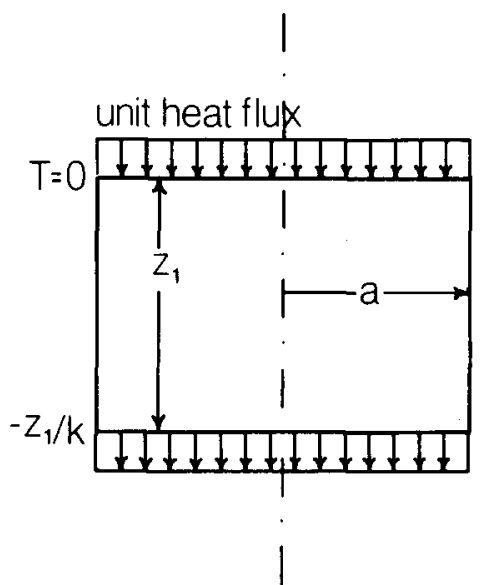

(a)

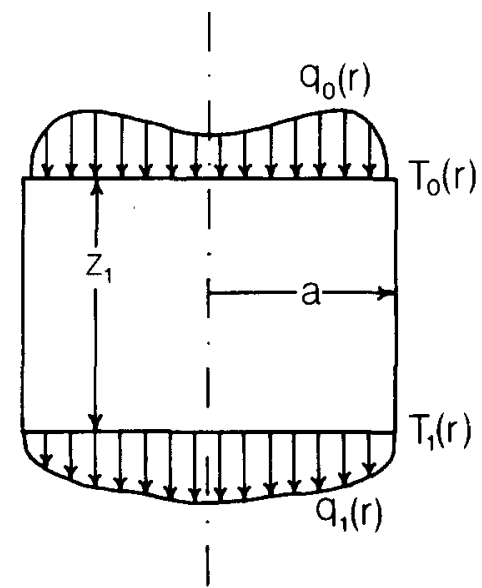

(b)

Figure 2. Solutions to be compared by the reciprocal theorem: (a) uniform heat flux; (b) arbitrary non-uniform heat flux.

The reciprocal theorem requires that

$$
\int_{S} T_{b} q_{a} \mathrm{~d} S=\int_{S} T_{a} q_{b} \mathrm{~d} S
$$

where the integrals are taken over the entire surface of the cylinders and $q_{a}, q_{b}$ are the fluxes normal to $S$ (taken as positive inwards).

The curved sides of the cylinders are insulated $\left(q_{a}, q_{b}=0\right)$, so the integrals only contain contributions from the end faces. We find

$$
\begin{aligned}
2 \pi & \int_{0}^{a} r T_{0}(r) \mathrm{d} r-2 \pi \int_{0}^{a} r T_{1}(R) \mathrm{d} r \\
& =2 \pi \int_{0}^{a} r \cdot \frac{z_{1}}{k} \cdot q_{1}(r) \mathrm{d} r \\
& =\frac{z_{1} Q}{k}
\end{aligned}
$$

from Eqn. (3).

Equation (18) applies for all values of $z$, and hence we can write

$$
\int_{0}^{a} T(r, z) \mathrm{d} r=-\frac{Q z}{2 \pi k}+C
$$

where

$$
C=\int_{0}^{a} r T_{0}(r) \mathrm{d} r
$$

is a constant.

From Eqn. (11), the tractions implied by the solution (4) are

$$
\sigma_{r r}=\frac{2 \alpha \mu(1+\nu)}{a^{2}}\left(\frac{Q z}{2 \pi k}-C\right), \quad r=a
$$


and they can be suppressed by superposing the state of biaxial hydrostatic bending and tension defined by

$$
\begin{aligned}
& \sigma_{r r}=\sigma_{\theta \theta}=-\frac{2 \alpha \mu(1+\nu)}{a^{2}}\left(\frac{Q z}{2 \pi k}-C\right), \\
& \sigma_{z z}=\sigma_{r \theta}=\sigma_{z \theta}=\sigma_{r z}=0 .
\end{aligned} \quad \text { all } r, \theta, z,
$$

The corresponding additional displacements are

$$
\begin{aligned}
& u_{r}=-\frac{\alpha(1-\nu) r}{a^{2}}\left(\frac{Q z}{2 \pi k}-C\right), \\
& u_{z}=\frac{\alpha(1-\nu) Q r^{2}}{4 \pi a^{2} k},
\end{aligned}
$$

omitting an arbitrary rigid body displacement.

\section{Distortion of the cylinder}

The distortion of the cylinder is best described in terms of the curvature of the generators $r=a$, and the plane faces $z=0, l$.

For any $z$ plane and particularly the end faces, the sum of the principal curvatures is

$$
\frac{\partial^{2} u_{z}}{\partial r^{2}}+\frac{1}{r} \frac{\partial u_{z}}{\partial r}=\frac{\alpha(1+\nu) q_{z}}{k}+\frac{\alpha(1-\nu) Q}{\pi a^{2} k}
$$

from Eqns. $(8,24)$, i.e.

$$
\frac{\partial^{2} u_{z}}{\partial r^{2}}+\frac{1}{r} \frac{\partial u_{z}}{\partial r}=\frac{\alpha}{k}\left\{(1+\nu) q_{z}+(1-\nu) \bar{q}\right\}
$$

where

$$
\bar{q}=Q / \pi a^{2}
$$

is the average heat flux through the cylinder.

The curvature of a generator of the cylinder, $r=a$, is

$$
\frac{\partial^{3} u_{r}}{\partial r \partial z^{2}}=-\frac{\partial^{3} \phi}{\partial r \partial z^{2}}
$$

by Eqns. $(4,23)$; and from Eqn. (5)

$$
\frac{\partial^{2} u_{r}}{\partial r \partial z^{2}}=-\alpha(1+\nu) \frac{\partial T}{\partial r}=0
$$

since the surface $r=a$ is insulated.

Thus, the generators remain straight and the cylinder deforms into a cone whose end faces are distorted in accordance with Eqn. (26).

In particular, we note that if the heat flux is uniform across the section $\left(q_{z}=\bar{q}\right)$, the right hand side of Eqn. (26) reduces to the constant $2 \alpha \bar{q} / k$ and the end planes become spherical - the heated plane convex and the cooled plane concave. This result is of course obtainable by more elementary means, noting that for uniform heat flux there are no induced thermal stresses. 


\section{Discussion}

Equation (26) shows that the curvature of the plane ends of the cylinder depends upon the local heat flux and the mean flux over the cross section. This contrasts with the result for the semi-infinite solid and the layer in which curvature depends upon local heat flux alone $[9,10]$. This difference has an important consequence when the direction of heat flow is into the least distortive material [11]. In this case, the mismatch of curvatures due to free expansion is such as to suggest that contact will be developed in an annular region, with a central circle of separation. This is the configuration assumed by Jones et al. [8] and it is supported by their experimental results. We note, however, that Eqn. (26) shows that their approximate treatment of the thermal distortion of the cylinders will become progressively less accurate as the contact region becomes smaller.

By contrast, if we consider the contact of a flat rigid punch with a half space [12], the relation between curvature and local heat flux rules out a solution with a central separation region. Instead we find that a central region of "imperfect thermal contact" [13] is developed. A similar configuration involving imperfect contact must be developed in the two-dimensional problem of two strips loaded on their plane ends, since in two-dimensional problems, the relation between local curvature and heat flux applies for simply connected bodies of any shape [11]. We also note that although the result of Eqn. (26) argues the possibility of a central separation region for the two cylinder problem with the appropriate direction of heat flow, an asymptotic analysis of the transition between perfect thermal contact and separation [14] shows that an intervening annulus of imperfect contact will also be required. However, experience with related problems $[13,15]$ suggests that this region will generally be small.

\section{Acknowledgements}

The author gratefully acknowledges support from the U.S. Army under contract no. DAAG29-82-K-0001.

\section{References}

[1] M.E. Barzelay, K.E. Tong and G.F. Holloway, Effect of pressure on thermal conductance of contact joints, NACA, TN 3295 (1955).

[2] M.G. Cooper, B.B. Mikic, and M.M. Yovanovich, Thermal contact conductance, Int. J. Heat Mass Transfer 12 (1969) 279-300.

[3] A.M. Clausing and B.T. Chao, Thermal contact resistance in a vacuum environment, Trans. A.S.M.E. 87C (1965) 243-251.

[4] A.M. Clausing, Heat transfer at the interface of dissimilar metals - the influence of thermal strain, Int. J. Heat Mass Transfer 9 (1966) 791-801.

[5] D.V. Lewis and H.C. Perkins, Heat transfer at the interface of stainless steel and aluminium - the influence of surface conditions on the directional effect, Int. J. Heut Mass Transfer 11 (1968) 1371-1383.

[6] T.R. Thomas and S.D. Probert, Thermal contact resistance - the directional effect and other problems, Int. J. Heat Mass Transfer 13 (1970) 789-807.

[7] J.R. Barber, The effect of thermal distortion on constriction resistance, Int. J. Heat Mass Transfer 14 (1971) 751-766.

[8] A.M. Jones, P.W. O'Callaghan and S.D. Probert, Thermal rectification due to distortions induced by heat fluxes across contacts between smooth surfaces, J. Mech Eng. Sci. 17 (1975) 252-261. 
[9] J.R. Barber, Thermoelastic contact problems, in A.D. DePater and J.J. Kalker (eds), The mechanics of the contact between deformable bodies. Delft University Press, 1975, pp. 171-190.

[10] J.R. Barber, Some implications of Dundurs' Theorem for thermoelastic contact and crack problems, $J$. Mech, Eng. Sci. 22 (1980) 229-232.

[11] J. Dundurs, Distortion of a body caused by free thermal expansion, Mech. Res. Comm. 1 (1974) 121-124.

[12] J.R. Barber, Indentation of an elastic half-space by a cooled flat punch, Q. J. Mech. Appl. Math. 35 (1982) 141-154.

[13] J.R. Barber, Contact problems involving a cooled punch, J. Elasticity 8 (1978) 409-423.

[14] M. Comninou and J. Dundurs, On the Barber boundary conditions for thermoelastic contact, A.S.M.E., J. Appl. Mech. 46 (1979) 849-853.

[15] M. Comninou, J. Dundurs and J.R. Barber, Planar Hertz contact with heat conduction, J. Appl. Mech. 47 (1980) 871-874. 